\title{
SUNRISE Chromospheric Infrared spectroPolarimeter (SCIP) for SUNRISE III: Scan mirror mechanism
}

Oba, Takayoshi, Shimizu, Toshifumi, Katsukawa, Yukio, Kubo, Masahito, Uraguchi, Fumihiro, et al.

Takayoshi Oba, Toshifumi Shimizu, Yukio Katsukawa, Masahito Kubo, Fumihiro Uraguchi, Toshihiro Tsuzuki, Tomonori Tamura, Kazuya Shinoda, Kazuhide Kodeki, Kazuhiko Fukushima, Achim Gandorfer, Jose Carlos del Toro Iniesta, "SUNRISE Chromospheric Infrared spectroPolarimeter (SCIP) for SUNRISE III: Scan mirror mechanism," Proc. SPIE 11445, Ground-based and Airborne Telescopes VIII, 114454F (13 December 2020); doi: $10.1117 / 12.2562075$ 


\title{
SUNRISE Chromospheric Infrared spectroPolarimeter (SCIP) for SUNRISE III: Scan Mirror Mechanism
}

\author{
Takayoshi Oba*a, Toshifumi Shimizu ${ }^{a}$, Yukio Katsukawa ${ }^{b}$, Masahito Kubo $^{\mathrm{b}}$, Fumihiro Uraguchi ${ }^{\mathrm{b}}$, \\ Toshihiro Tsuzuki ${ }^{b}$, Tomonori Tamura ${ }^{b}$, Kazuya Shinoda ${ }^{b}$, Kazuhide Kodeki ${ }^{\mathrm{c}}$, Kazuhiko \\ Fukushima $^{\mathrm{c}}$, Achim Gandorfer ${ }^{\mathrm{d}}$, Jose Carlos del Toro Iniesta ${ }^{\mathrm{e}}$ \\ ${ }^{a}$ Institute of Space and Astronautical Science, Japan Aerospace Exploration Agency, 3-1-1 \\ Yoshinodai, Chuou, Sagamihara, Kanagawa 252-5210, Japan \\ b National Astronomical Observatory of Japan, 2-21-1 Osawa, Mitaka, Tokyo 181-8588, Japan \\ ${ }^{c}$ Mitsubishi Electric Corporation, 8-1-1 Tsukaguchihonmachi, Amagasaki, Hyogo 661-8661, Japan \\ d Max-Planck-Institut für Sonnensystemforschung, Justus-von-Liebig-Weg 3, 37077 Göttingen, \\ Germany

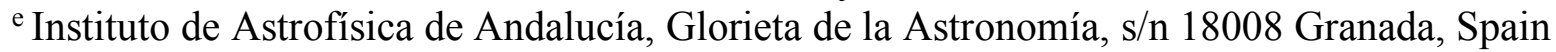

\begin{abstract}
The SUNRISE Chromospheric Infrared spectroPolarimeter (SCIP) is a balloon-borne long-slit spectrograph for SUNRISE III to precisely measure magnetic fields in the solar atmosphere. The scan mirror mechanism (SMM) is installed in the optical path to the entrance slit of the SCIP to move solar images focused on the slit for 2-dimensional mapping. The SMM is required to have (1) the tilt stability better than $0.035^{\prime \prime}(3 \sigma)$ on the sky angle for the diffraction-limited spatial resolution of $0.2^{\prime \prime}$, (2) step response shorter than $32 \mathrm{msec}$ for rapid scanning observations, and (3) good linearity (i.e. step uniformity) over the entire field-of-view ( $\left.60^{\prime \prime} \times 60^{\prime \prime}\right)$. To achieve these performances, we have developed a flight-model mechanism and its electronics, in which the mirror tilt is controlled by electromagnetic actuators with a closed-loop feedback logic with tilt angles from gap-based capacitance sensors. Several optical measurements on the optical bench verified that the mechanism meets the requirements. In particular, the tilt stability achives better than $0.012^{\prime \prime}(3 \sigma)$. Thermal cycling and thermal vacuum tests have been completed to demonstrate the performance in the vacuum and the operational temperature range expected in the balloon flight. We found a small temperature dependence in the step uniformity and this dependence will be corrected to have 2-demensional maps with the sub-arcsec spatial accuracy in the data post-processing.
\end{abstract}

Keywords: SUNRISE III, Balloons, Sun, SCIP, Tip-tilt mirror, Spectro-polarimeter

\section{INTRODUCTION}

Spectroscopic and spectro-polarimetric observations are necessarily to diagnose the physical quantities of astrophysical phenomena, such as temperature, velocity, and magnetic field. Slit observation system with spectroscopy and spectropolarimetry disperses one-dimensional light passing through the slit into the wavelength direction, and then the resultant two-dimensional (spatial and spectral) light are fed to the detector. To compose a map in the two spatial dimensions, each pixel of which includes spectroscopic (spectro-polarimetric) information, the scan mirror mechanism (SMM) tilts a mirror and moves the image on the slit.

We have developed the SMM that is for a slit-scan system of the SCIP (SUNRISE Chromospheric Infrared spectroPolarimeter), onboard the international joint balloon project SUNRISE III, which is to be launched on 2022. This balloon project has experienced two flights in $2009^{1}$ and $2013^{2}$, by an international collaboration among Germany, Spain, and United States, and Japan is joining the project in the third flight. The prime aperture of this balloon-borne telescope is $1 \mathrm{~m}$, which is the largest size so far in a solar balloon or a solar space mission. Reaching at an altitude of 35-37 km in the strato-

*oba.takayoshi@jaxa.jp; phone +81-50-3362-6949 
sphere, the balloon-borne telescope provides spectropolarimetric data with high spatial resolution and high accuracy under a lack of seeing-induced image degradation. Launched from ESRANGE near KIRUNA/Sweden, the balloon flies to Canada over the Atlantic Ocean with flight duration of approximately 1 week. Japan has developed the SCIP ${ }^{3}$ that observes two near-infrared bands of $770 \mathrm{~nm}$ and $850 \mathrm{~nm}$ broadly in wavelength by a slit-scan system, in order to diagnose the physical quantities seamlessly from the solar photosphere to the solar chromosphere ${ }^{4}{ }^{5}$. The SCIP achieves the diffractionlimited spatial resolution of $0.2^{\prime \prime}$ in the balloon-flight environment, which is achieved with the optimized bench material by newly designing the opto-mechanics ${ }^{6}$. Since magnetic elements in the chromosphere consist of broad spatial scales and they behave highly dynamic, the SMM needs to scan rapidly and widely with high accuracy. To meet such requirements, we have developed the SMM in which the mirror tilt is controlled by electro-magnetic actuators with sensing a tilt by gapbased capacitance sensors. The SMM should be verified its performances under the flight environment, i.e., low/high temperature together with low pressure.

\section{SMM (SCAN MIRROR MECHANISM)}

\subsection{General description}

The SMM consists of the three components; SMM-TM (Tip-tilt Mirror), SMM-DRV (Driver), and SMM-IF (Interface). Figure 1 shows photography the outside appearance of the SMM components. Figure 2 describes the block diagram together with the related components in communication. The $\mathrm{PMU}^{7}$ (Polarization Modulation Unit) rotates a waveplate continuously at a constant speed, installed in the SCIP. The PMU sends periodically a synchronization signal to the SCIP-E (Electronics). The SCIP-E receives the synchronization signal and then transmits a tip-tilt command to the SMM-DRV, thereby controlling a tilt of the mirror. The role of the SMM-IF is an amplifier of signals output by the gap-sensors. The SMMDRV transmits HK (Housekeeping) data, e.g., temperatures of each component and mirror angles, to the SCIP-E. The SMM-TM, installed in the Image Stabilization and Light Distribution ${ }^{8}$ (ISLiD), feeds the light collected from the primary and secondary mirrors onto the entrance slit of the SCIP by tilting the mirror.

Figure 3 is a schematic drawing of the SMM-TM. The electromagnetic attraction system is adopted for the tilt drive mechanism. The prime requirement in selecting this system is a broad range of mirror tilting with high mechanical strength, for the future application to space-borne telescopes. The SMM-DRV controls the current that flows into electromagnetic actuators (coils), and the attracting force acts on the magnetic plate mounted behind the mirror. The four actuators control the mirror tilt in the two-dimension ( $\theta \mathrm{x}, \theta \mathrm{y}$ ). Capacitance gap sensors (CPL230, Lion Precision) are installed to the SMMTM in order to detect a tilt of the mirror, by precisely measuring a distance of the gap. The two sensors are arranged in the one-dimensional direction such that the mirror angle of $\theta \mathrm{x}$ is derived by addition of outputs in the two gap sensors and the mirror angle of $\theta \mathrm{y}$ is derived by subtraction. The detected mirror angles are fed back for a determination of the strength of the current that flows into the four actuators. The mirror tilt is controlled with high precision by such closed-loop system. Since the scan direction of the SCIP is set to the $\theta y$ direction perpendicular to the slit direction, we focus on evaluation of the performances of the scan in the $\theta y$ direction.
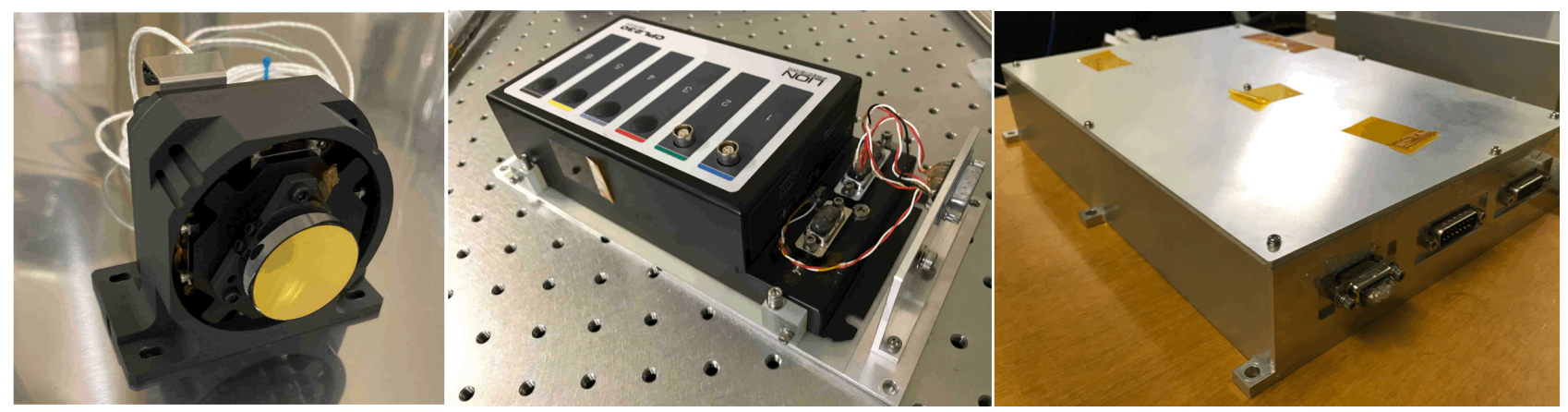

Figure 1: Photographs of the SMM-TM, SMM-IF, and SMM-DRV, from left to right panel. 


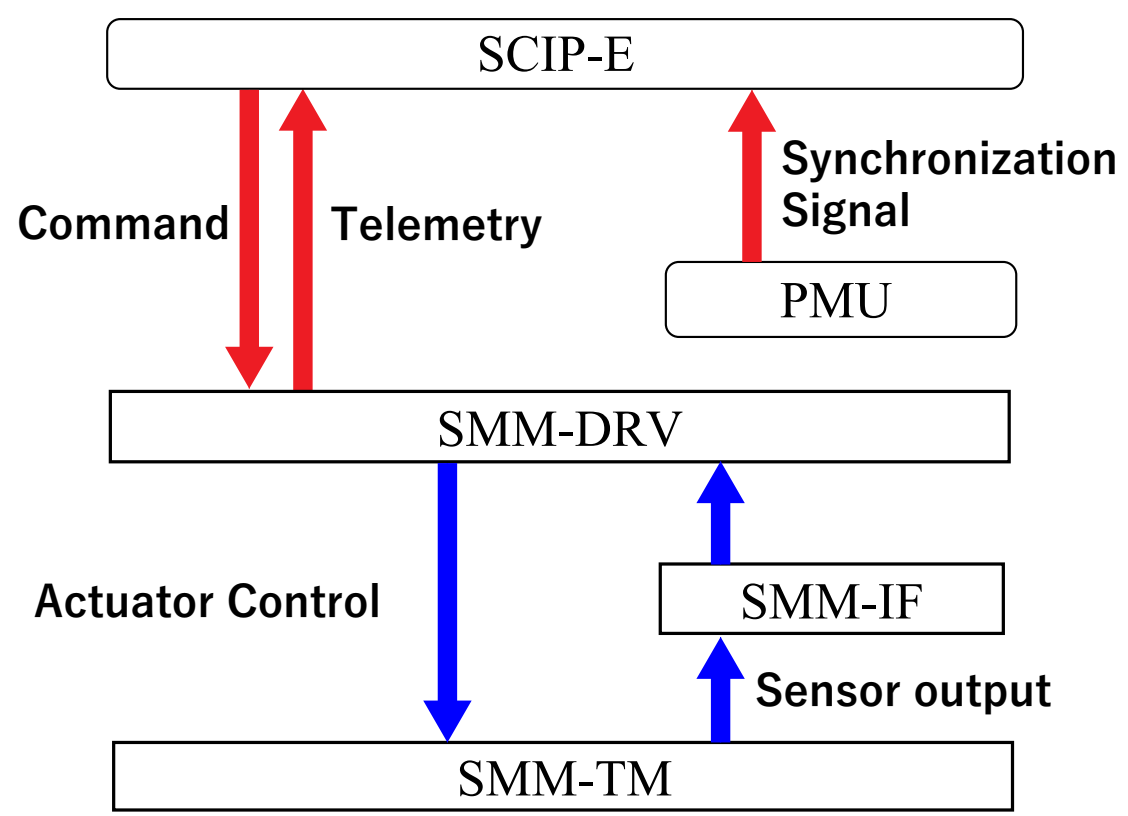

Figure 2: Block diagram between the SMM components together with the related components in communication. Arrow indicates a direction of signal. Red and blue colors mean digital and analog signals, respectively.

[A]

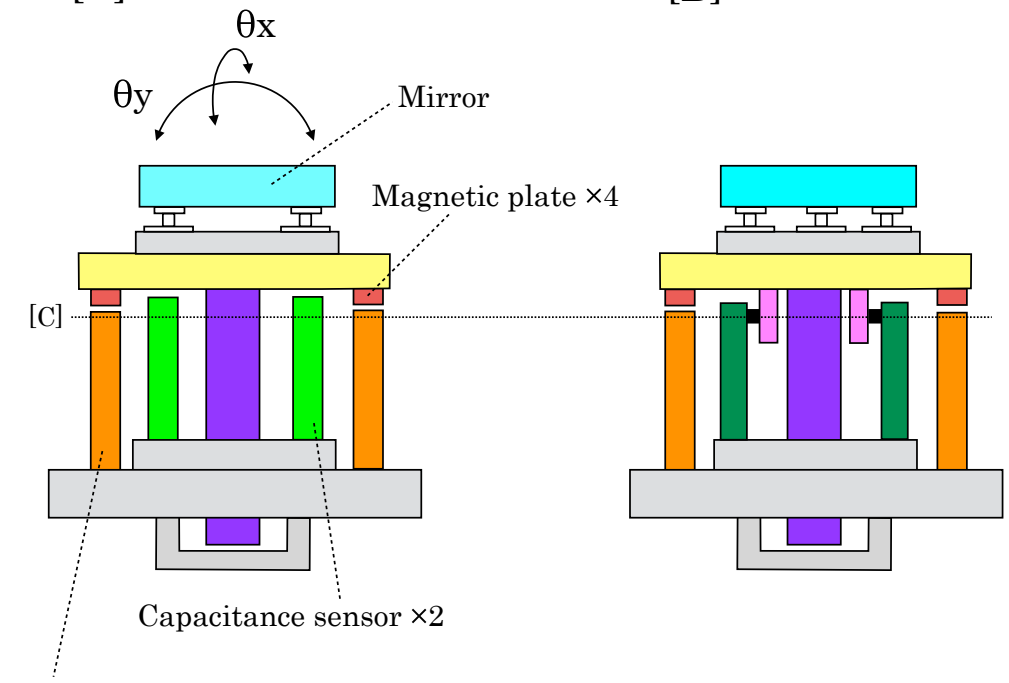

Electromagnetic actuator $\times 4$

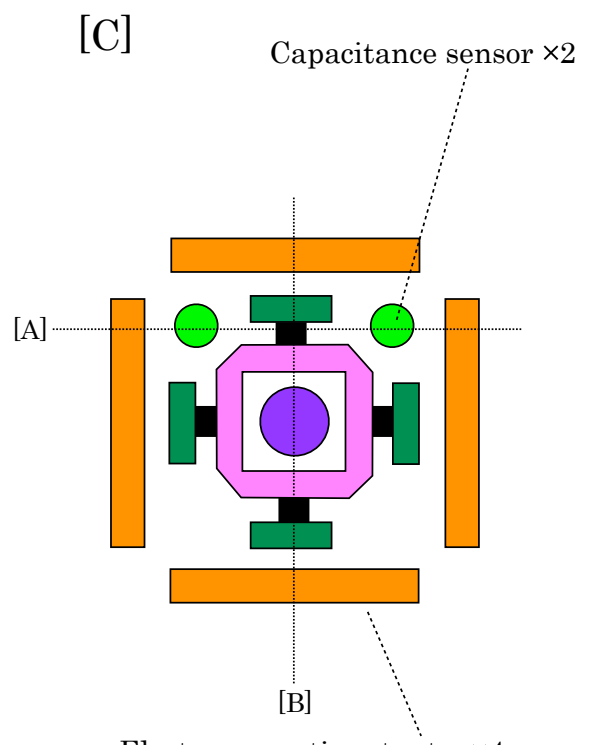

Electromagnetic actuator $\times 4$

Figure 3: Cross sections cutting through the three parts. Dotted lines indicate the sections of A, B, and C.

\subsection{Scan function}

Figure 4 describes a schematic overview of relation in time among the commands and tip-tilt mirror and exposure time of the camera in the SCIP during the fastest scan mode (64 msec is allocated for one step). The SCIP-E transmits a tip tilt command at the timing of receiving synchronization signals sent from the PMU every $32 \mathrm{msec}$. An exposure time of the camera starts $32 \mathrm{msec}$ after receiving a tip-tilt command (no exposure time are within $32 \mathrm{msec}$ of receiving the tip-tilt command). HK data is transmitted to the SCIP-E from the SMM-DRV every $32 \mathrm{msec}$. 


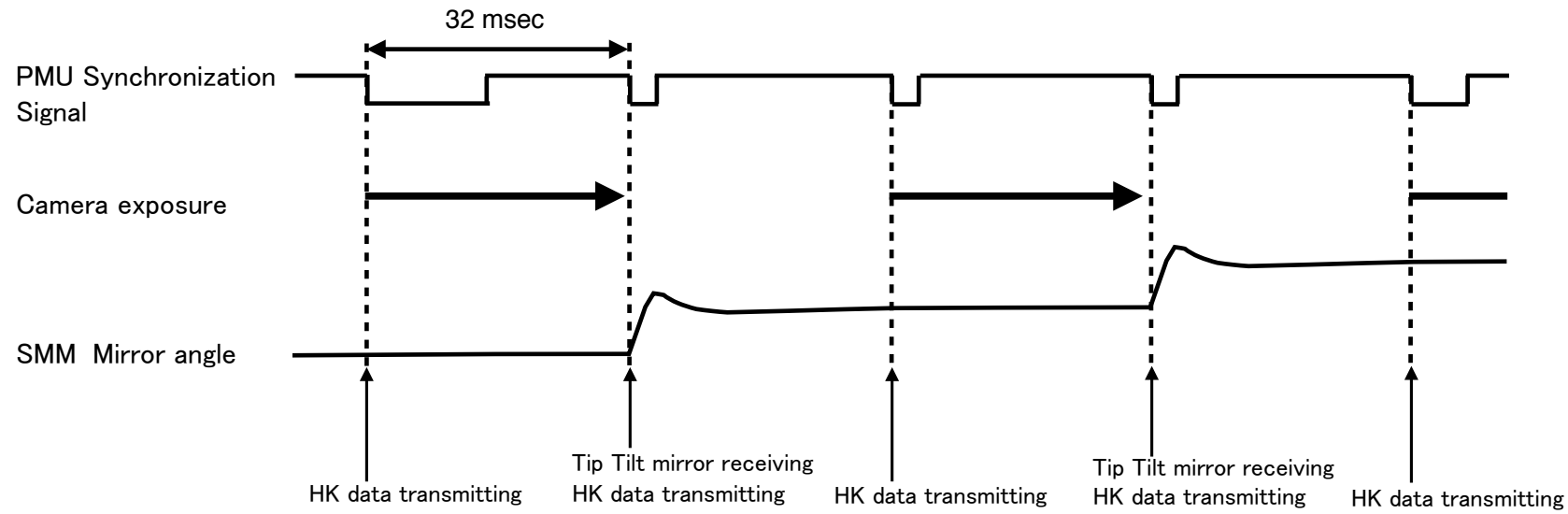

Figure 4: Overview of the relation between the commands (transmission of HK data and reception of tip-tilt mirror command) and exposure time of the camera during the fastest scan.

\subsection{Requirements}

The requirements to the SMM is summarized in Table 1. The step resolution $\left(0.0936^{\prime \prime}\right)$ corresponds to the slit width of the SCIP. The drive range $\left( \pm 33^{\prime \prime}\right)$ exceeds sufficiently the field-of-view (FoV) of $\pm 29^{\prime \prime}$. The stability $\left(<0.035^{\prime \prime}\right)$ is allocated for the SMM in order to obtain images with the diffraction-limited resolution of $0.2^{\prime \prime \prime}$. The linearity error $(<0.2 \%)$ corresponds to an offset of one step ( $\left.0.0936^{\prime \prime}\right)$ divided by the end of scan position (33"), i.e., $0.0936^{\prime \prime} / 33^{\prime \prime}=0.0028$. The specified values on mechanical angle in Table 1 are converted to those on sky angle through a multiplication by the optical magnification. The step response of $32 \mathrm{msec}$ corresponds to the time interval between when receiving the tip tilt command and when reaching the position at the next scan step (Figure 4). Survival and operational temperatures cover an in-flight temperature range.

Table 1. Performances of the SMM

\begin{tabular}{|l|l|}
\hline \multicolumn{1}{|c|}{ Items } & \multicolumn{1}{c|}{ Specification } \\
\hline Step resolution & $\begin{array}{l}2.857^{\prime \prime} \text { on mechanical angle } \\
\left(0.0936^{\prime \prime} \text { on sky angle }\right)\end{array}$ \\
\hline Drive range & $\begin{array}{l} \pm 1006^{\prime \prime} \text { on mechanical angle } \\
\left( \pm 33^{\prime \prime} \text { on sky angle }\right)\end{array}$ \\
\hline Step response & $<32 \mathrm{msec}$ \\
\hline Stability & $\begin{array}{l}1^{\prime \prime}[3 \sigma] \text { on mechanical angle } \\
\left(0.035^{\prime \prime}[3 \sigma] \text { on sky angle }\right)\end{array}$ \\
\hline Linearity error & $<0.2 \%$ \\
\hline Pressure & $<133 \mathrm{~Pa}(\sim 1$ Torr $)$ \\
\hline Survival temperature & $\begin{array}{l}\text { SMM-TM } \cdot \text { SMM-IF: }-10 \sim 50{ }^{\circ} \mathrm{C} \\
\text { SMM-DRV: }-20 \sim+50{ }^{\circ} \mathrm{C}\end{array}$ \\
\hline Operational Temperature & $\begin{array}{l}\text { SMM-TM } \cdot \text { SMM-IF: }+10 \sim+45^{\circ} \mathrm{C} \\
\text { SMM-DRV: } 0 \sim+45^{\circ} \mathrm{C}\end{array}$ \\
\hline
\end{tabular}




\section{BASIC FUNCTIONAL TESTS}

\subsection{Linearity}

We evaluated the linearity of an actual mirror position with respect to a commanded angle, to validate the mirror angle can be precisely positioned over the range of the full FoV. The optoelectric autocollimator (TAUS300-57, TRIOPTICS) measures a tilt angle of the SMM-TM, set to be confronted with the SMM-TM on the optical bench.

The autocollimator measures a total step of 705 with a uniform interval of 2.857" in commanding, covering the full FoV of 1005.66". In Figure 5, the left panel displays the measured angles with respect to the commanded angles, and the right panel displays the residual. The residual is calculated by the subtraction of a commanded angle from a measured angle. The linear coefficient used for an evaluation of the linearity is defined as

$$
y_{f i t}=a y_{\text {command }}+b
$$

where $a$ is a linear coefficient, $y_{\text {command }}$ is a commanded angle, $b$ is offset with respect to $0^{\prime \prime}$, and $y_{f i t}$ is the fitting function for the measured angles. The linearity error is introduced by

$$
\text { Linearity error }=\operatorname{Max}\left(\frac{\left|y_{\text {measured }}-y_{\text {fit }}\right|}{1005.66 \times 2} \times 100\right)
$$

where $y_{\text {measured }}$ is a measured angle. The measured angles in Figure 5 is fitted with Equation (1), which provides $a=1.0041$ and $b=0.91$. This value of the linear coefficient indicates that the scanned map expands by $0.41 \%$. Clearly seen from the right panel of Figure 5, the step interval is not constant and it varies periodically over a scale of $\sim 500 "$. The linearity error is $0.08 \%$, which is lower than the requirement $(<0.2 \%)$.

We investigated the temperature dependence of the linear coefficient by controlling the room temperature. The linear coefficient is correlated well with the temperature of the SMM-TM (Figure 6). High temperature induces a sparse scanstep (high linear coefficient), while lower temperature induces a dense scan-step (low linear coefficient). This temperature dependence of the step interval would be caused by the thermal expansion of the SMM-TM material and temperaturedependent signal output by the capacitance gap sensors. For the flight data, a post processing would correct for a sparse or dense step interval according to the SMM-TM temperature recorded throughout the flight, interpolating from the tight correlation drawn by the grey line in the right panel of Figure 6.
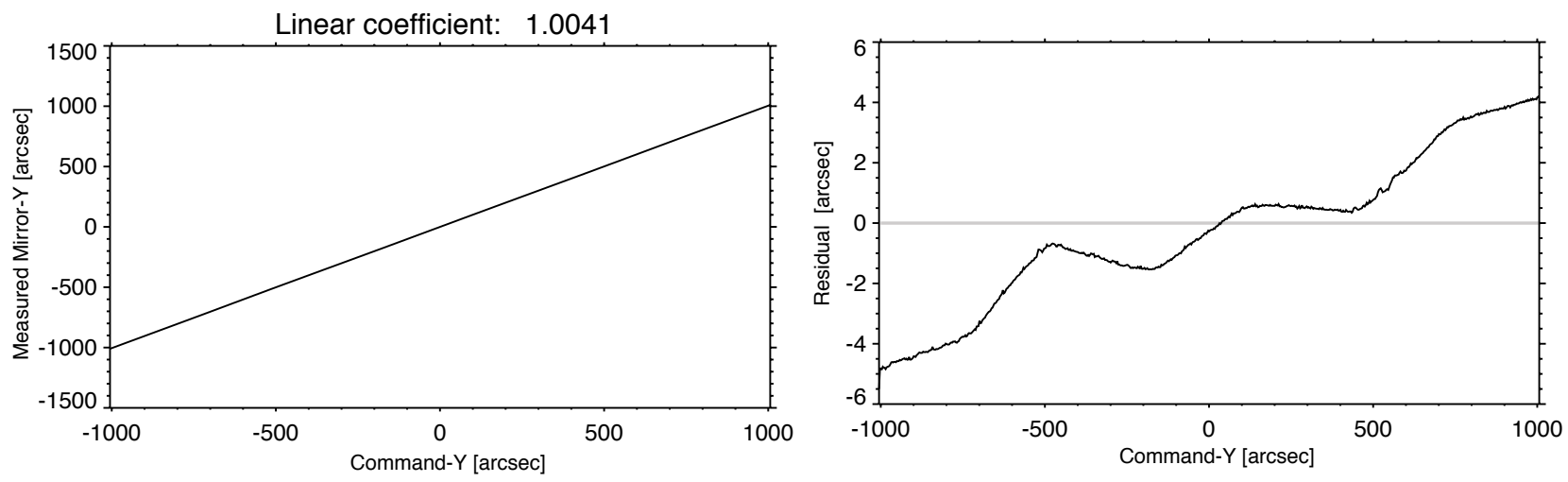

Figure 5: Left: Measured angle as a function of a commanded angle. Right: Residual, calculated by the subtraction of a commanded angle from a measured angle, as a function of a commanded angle. Units are mechanical angle. 

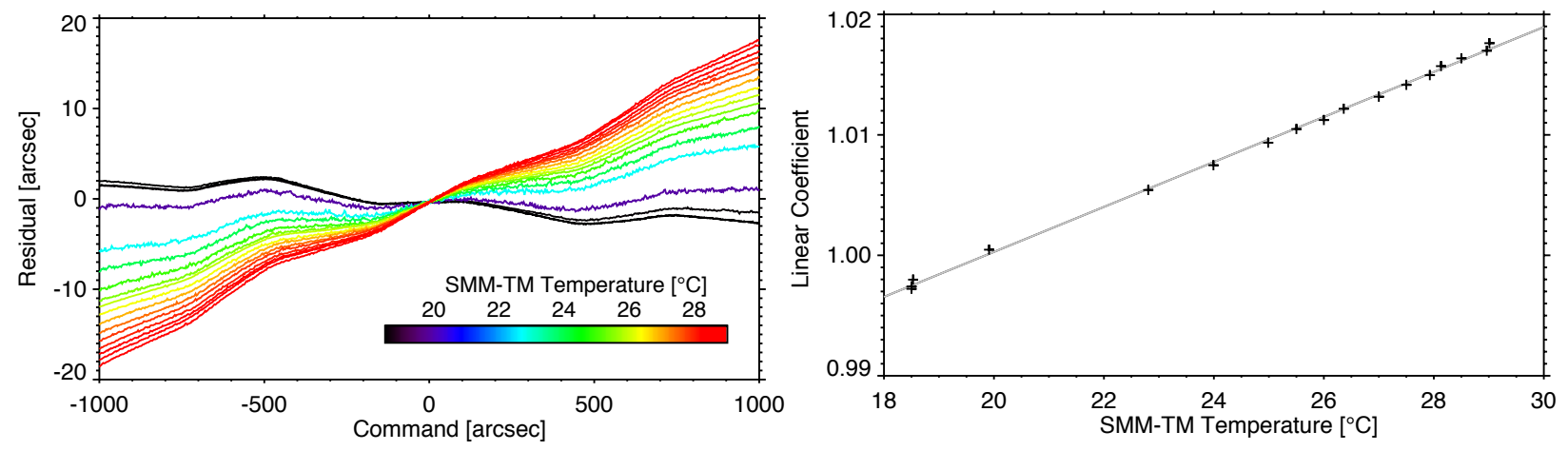

Figure 6: Left: SMM-TM temperature dependence of the residual. Unit is mechanical angle. Right: Linear coefficient as a function of the SMM-TM temperature together with the best fitted linear function in grey.

\subsection{Stability}

We evaluated the stability of the SMM-TM by the optical measurement of the mirror jittering through the afocal system (Figure 7). In this optical system, the beam emitted from the laser becomes a diverging ray after passing through the spatial filter. Then, the ray becomes a collimator beam by passing through the lens 1 , and is reflected by the mirror of the SMMTM. Finally, the beam is condensed by the lens 2 and imaged on the detector with the offset, displaced correspondingly by a tip-tilt angle of the SMM-TM. The PSD (Position Sensitive Detector) S1880 is used for the detector. The sampling rate of the PSD is set to $1 \mathrm{kHz}$.

Figure 8 shows jitters in the $\theta \mathrm{x} \cdot \theta \mathrm{y}$ directions at the $\left[0^{\prime \prime}, 0^{\prime \prime}\right]$. Jitters, defined by $3 \sigma$ where $\sigma$ is standard deviation, are $0.10^{\prime \prime}$ and $0.09^{\prime \prime}$ on mechanical angles in the $\theta \mathrm{x}$ and $\theta \mathrm{y}$ directions, respectively. These values are sufficiently lower than the requirement of $3 \sigma<1$ " (Table 1). Jitters at the other mirror angles are summarized in Table 2. The jitters over the FoV fall into a range of $0.08^{\prime \prime}-0.10^{\prime \prime}$ on mechanical angle and are independent of the $\mathrm{FoV}$, which is also significantly lower than the requirement of $3 \sigma<1 "$.

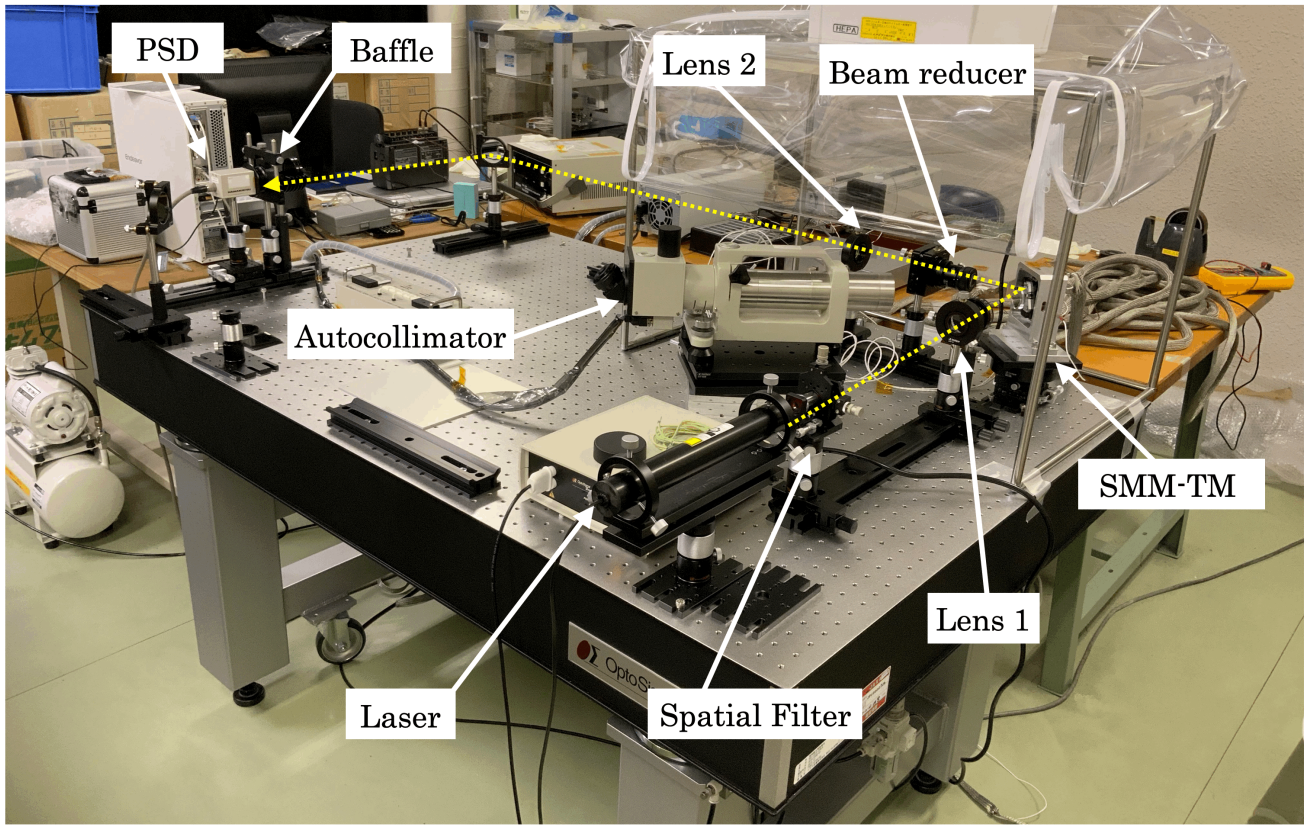

Figure 7: Optical measurement configuration for jitter and step response measurements. 

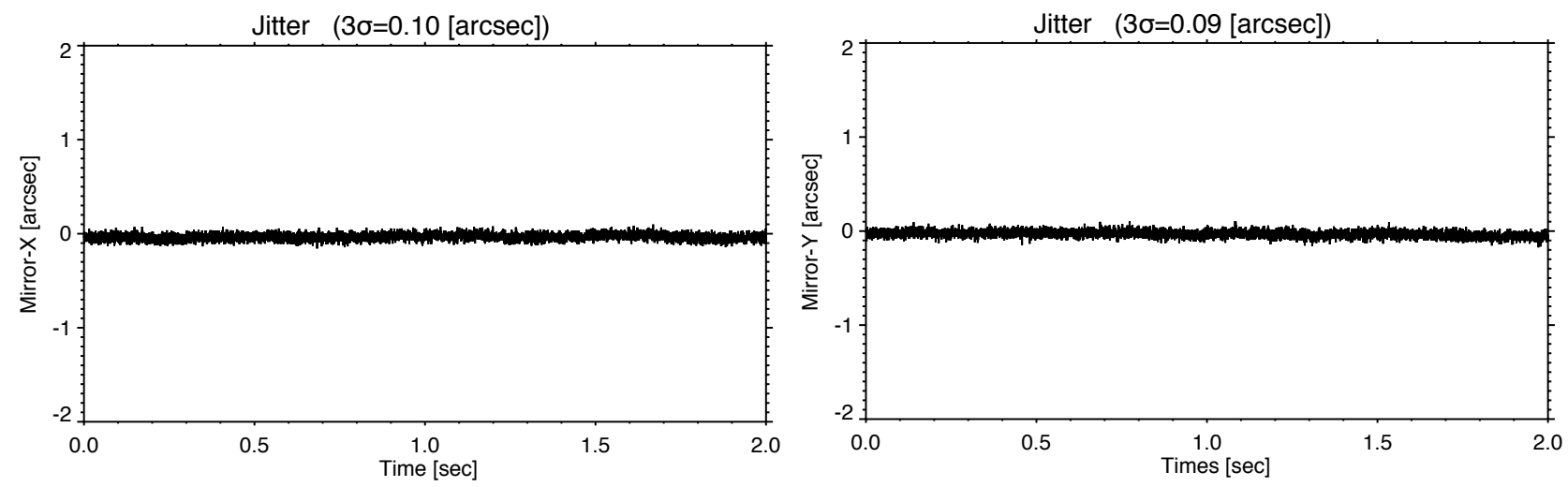

Figure 8: Mirror-X and Mirror-Y angles in left and right panels, respectively. Units are mechanical angle.

Table 2: Jitter $(3 \sigma)$ over the FoV. Left and right values in each cell denote Mirror-X and Mirror-Y angles, respectively. Units are mechanical angle.

\begin{tabular}{|c|c|c|c|}
\hline & $\theta \mathrm{x}=-201^{\prime \prime}$ & $\theta \mathrm{x}=0^{\prime \prime}$ & $\theta \mathrm{x}=+201^{\prime \prime}$ \\
\hline$\theta \mathrm{y}=+793^{\prime \prime}$ & $0.10^{\prime \prime}, 0.08^{\prime \prime}$ & $0.09^{\prime \prime}, 0.09^{\prime \prime}$ & $0.09^{\prime \prime}, 0.09^{\prime \prime}$ \\
\hline$\theta \mathrm{y}=00^{\prime \prime}$ & $0.09^{\prime \prime}, 0.09^{\prime \prime}$ & $0.10^{\prime \prime}, 0.09^{\prime \prime}$ & $0.08^{\prime \prime}, 0.09^{\prime \prime}$ \\
\hline$\theta \mathrm{y}=-793^{\prime \prime}$ & $0.09^{\prime \prime}, 0.09^{\prime \prime}$ & $0.09^{\prime \prime}, 0.09^{\prime \prime}$ & $0.09^{\prime \prime}, 0.09^{\prime \prime}$ \\
\hline
\end{tabular}

\subsection{Step response}

In the step response test, the optical measurement configuration is same as the stability test (Figure 7). Figure 9 shows the mirror movement during the fastest scan mode (Figure 4). The mirror position reaches the local maximum 3 msec after receiving the tip-tilt command, and subsequently reaches the local minimum $10 \mathrm{msec}$ after receiving the command, and gradually approaches the next scan-step position. Since an exposure time starts $32 \mathrm{msec}$ after receiving the tip-tilt command, the arrival time of the next step position should be less than $32 \mathrm{msec}$. In the analysis, the arrival time is determined by when the mirror reaches 2.757 ", set by the subtraction of $0.1^{\prime \prime}$ from the scan-step of $2.857^{\prime \prime}$. We obtained the step response of $26.1 \mathrm{msec}$, which meets the requirement of $<32 \mathrm{msec}$.

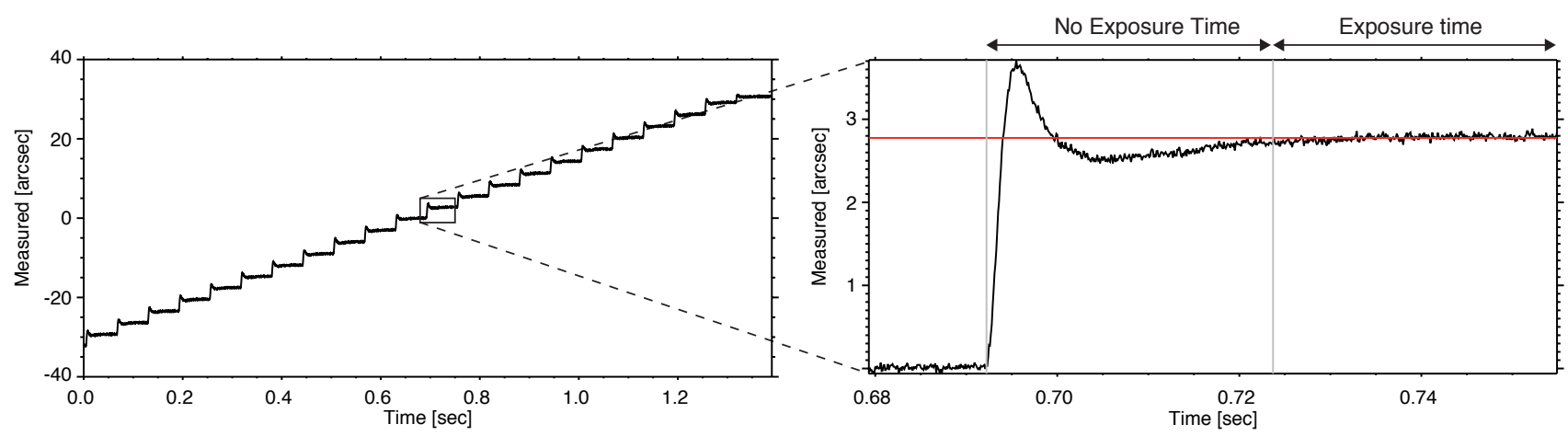

Figure 9: Left) Mirror-Y angle during the fastest scan mode. Right) Enlarged view of the Mirror-Y angle for one step. Start time of the mirror movement and of the camera exposure are indicated by the vertical grey lines, in left and right, respectively. The next step position is marked with the horizontal red line. Units are mechanical angle. 


\section{THERMAL CYCLING TEST}

In the balloon flight, heat load due to a variation of the ambient temperature could cause an equipment failure. The thermal cycling test is to verify that the SMM can exert its scan function without any functional problems after a head load. In NAOJ / Advanced Technology Center, the SMM components were placed inside the thermal chamber (Figure 10), and loaded with a thermal variation. Since the survival/operational temperatures of the SMM-DRV are different from those of the SMM-TM/SMM-IF (Table 1), the test has been performed separately with a different temperature variation; the upper and the lower temperature limits are $50^{\circ} \mathrm{C}$ and $-20^{\circ} \mathrm{C}$ for the SMM-DRV, while are $50^{\circ} \mathrm{C}$ and $-10^{\circ} \mathrm{C}$ for the SMM-TM and the SMM-IF. Total of 5 cycles of the temperature variation is distributed into 2 cycles at $1^{\text {st }}$ day and 3 cycles at $2^{\text {nd }}$ day (Figure 11). After the heat load, we turned on the SMM and exert a scan function at the temperatures of the upper and lower operational limits. HK data recorded in the test verified that the mirror could tilt at the commanded angles during the scan without any functional failures.
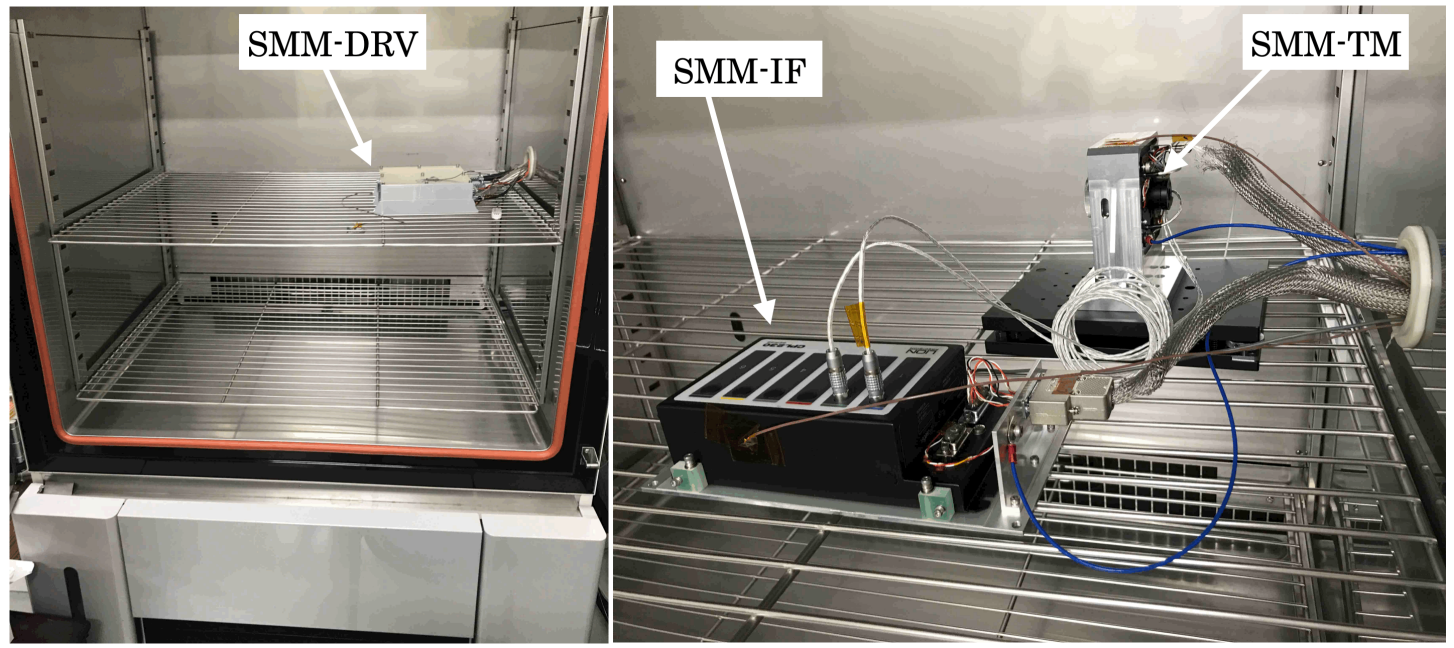

Figure 10: Left) SMM-DRV placed inside the thermal chamber. Right) SMM-TM and SMM-IF placed inside the thermal chamber.
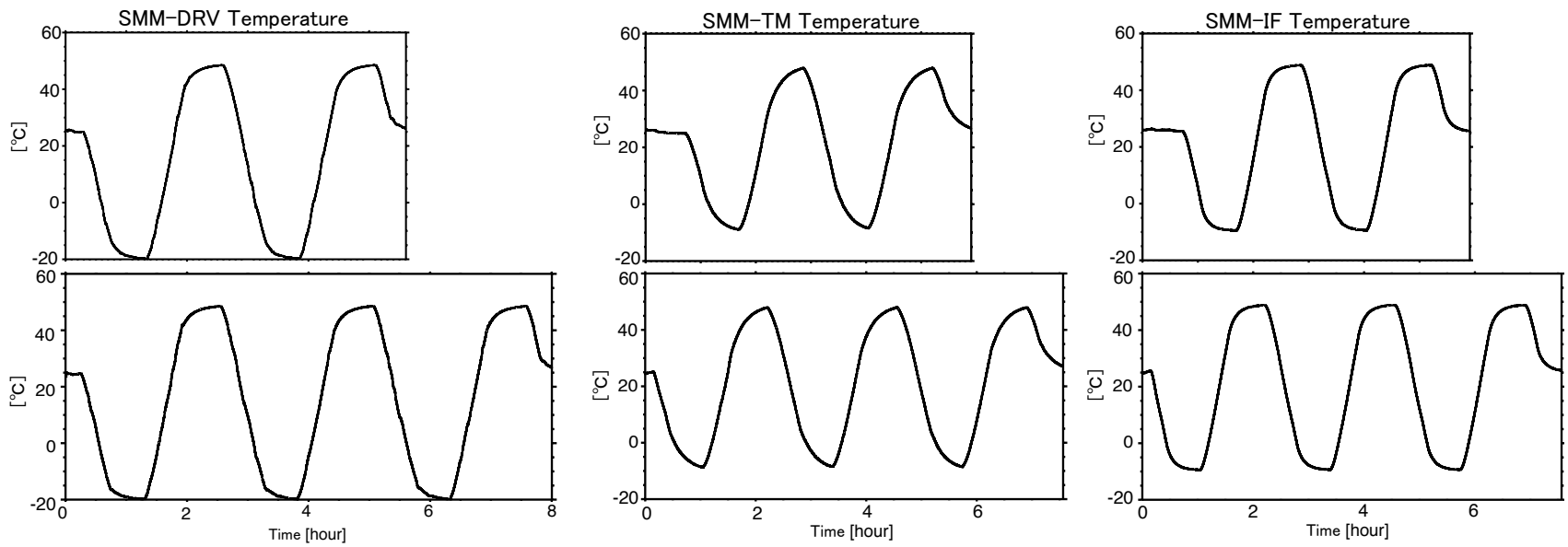

Figure 11: Temperature variation loaded on the SMM-DRV, the SMM-TM, and the SMM-IF, from left to right. 2 cycles and 3 cycles of the variation are loaded at $1^{\text {st }}$ and $2^{\text {nd }}$ day, plotted on the upper and lower rows, respectively. 


\section{THERMAL VACUUM TEST}

The thermal vacuum test is to verify that the performance of the SMM meets the requirement under the balloon-flight environments, such as low/high temperature with the vacuum condition. The SMM is placed inside a vacuum chamber (Figure 12), in which the pressure has been kept less than $10^{-2} \mathrm{~Pa}$. The temperature environment was controlled through the shroud set inside the vacuum chamber (seen as the reddish-brown envelope in the insert of Figure 12). We confirmed that the SMM could execute the scan function at temperatures of $10 \cdot 20 \cdot 25 \cdot 30 \cdot 40^{\circ} \mathrm{C}$. Another optoelectric autocollimator (PA102, Nikon), placed outside the vacuum chamber but confronted with the SMM-TM through a view port, measures a tilt angle of the SMM-TM. The temperature dependence of the linear coefficient is shown in the right panel of Figure 12. The linear coefficients over the temperature range of $20-30^{\circ} \mathrm{C}$ are compatible with those under the atmospheric pressure (Figure 6), and the coefficients are also on the linear correlation extending to $10^{\circ} \mathrm{C}$ and $40^{\circ} \mathrm{C}$. Jitters in $3 \sigma$ at temperature range of $10-40^{\circ} \mathrm{C}$, calculated from the HK data, are summarized in Table 3. Jitter does not depend on the SMM-TM temperature and is better than $0.41^{\prime \prime}$ on mechanical angle, which meets the requirement of $3 \sigma<1.0^{\prime \prime}$ $\left(0.41^{\prime \prime}\right.$ is converted to $0.012^{\prime \prime}$ on sky angle). Jitters in the thermal vacuum test is slightly higher than those in the optical measurements (Figure 8 and Table 2). We could ascribe this difference to the use of an optical bench, by comparison with the jitters calculated from the HK data using the optical bench (listed in the right-most of Table 3).
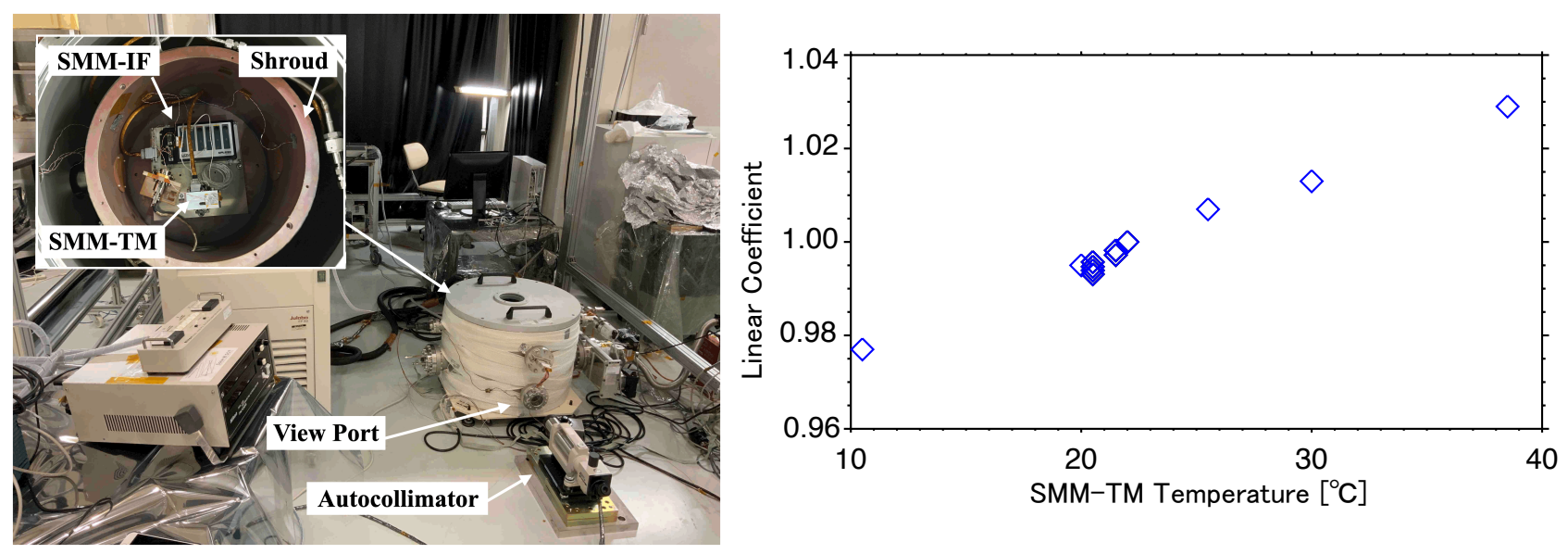

Figure 12. Left) Overview of the thermal vacuum test. Insert shows the inside of the vacuum chamber. Right) Linear coefficient as a function of the SMM-TM temperature.

Table 3: Jitter $(3 \sigma)$ at mirror angle of $\left[0^{\prime \prime}, 0^{\prime \prime}\right]$ for various experimental conditions, calculated from HK. Units are mechanical angle.

\begin{tabular}{|c|c|c|c|c|c|c|}
\hline \multirow{2}{*}{} & \multicolumn{4}{|c|}{ Thermal Vacuum Test (inside the vacuum chamber) } & \multirow{2}{*}{$\begin{array}{c}\text { Basic functional Test } \\
\text { (on the optical bench) }\end{array}$} \\
\cline { 2 - 6 } & $10^{\circ} \mathrm{C}$ & $20^{\circ} \mathrm{C}$ & $25^{\circ} \mathrm{C}$ & $30^{\circ} \mathrm{C}$ & $40^{\circ} \mathrm{C}$ & $0.11^{\prime \prime}$ \\
\hline$\theta \mathrm{x}$ & $0.21^{\prime \prime}$ & $0.12^{\prime \prime}$ & $0.30^{\prime \prime}$ & $0.15^{\prime \prime}$ & $0.15^{\prime \prime}$ & $0.13^{\prime \prime}$ \\
\hline$\theta \mathrm{y}$ & $0.15^{\prime \prime}$ & $0.41^{\prime \prime}$ & $0.18^{\prime \prime}$ & $0.26^{\prime \prime}$ & $0.28^{\prime \prime}$ & \\
\hline
\end{tabular}




\section{SUMMARY}

A series of the tests of the optical measurements, the thermal cycling, and the thermal vacuum tests, verified that the SMM achieves the requirements in the balloon flight environment. Spectroscopic measurements with a slit-scan system by SUNRISE III/SCIP would satisfactory provide a high-quality data. The slit-scan system is not only required by SUNRISE III/SCIP, but also necessary for the future solar space-borne telescope, Solar-C (EUVST), launched on the mid-2020s. The component technology developed in the SMM can be also applied to space-borne telescopes in the future.

\section{ACKNOWLEDGMENTS}

The SUNRISE III project is funded in Japan by the ISAS/JAXA Small Mission-of-Opportunity program for novel solar observations, JSPS KAKENHI Grant Number 18H05234 (PI:Y.Katsukawa), and NAOJ Research Coordination Committee, NINS. We would also thank significant technical support from the Advanced Technology Center (ATC), NAOJ.

\section{REFERENCES}

[1] Barthol, P., et al., “The Sunrise Mission”, Solar Physics 268, 1-34 (Jan. 2011).

[2] Solanki, S. K., et al., "The Second Flight of the SunRISE Balloon-borne Solar Observatory: Overview of Instrument Updates, the Flight, the Data, and First Results", The Astrophysical Journal Supplement Series, 229:2 (16pp), 2017 March

[3] Katsukawa, Y., et al., "SUNRISE Chromospheric Infrared spectroPolarimeter (SCIP) for SunRISE III: System Design and Capability", Ground-based and Airborne Instrumentation for Astronomy VIII, SPIE Astronomical Telescopes Instrumentation (2020)

[4] Quintero Noda, C., et al., "Chromospheric polarimetry through multiline observations of the 850-nm spectral regionII. a magnetic flux tube scenario", Monthly Notices of the Royal Astronomical Society 472 (1), 727-737 2017.

[5] Quintero Noda, C., et al., "Solar polarimetry in the $\mathrm{K} \mathrm{ID}_{2}$ line: A novel possibility for a stratospheric balloon", Astronomy \& Astrophysics 610, A79 (Mar. 2018).

[6] Uraguchi, F., et al., "SUNRISE chromospheric infrared spectropolarimeter (SCIP) for SUNRISE III: Opto-mechanical analysis and design", Ground-based and Airborne Instrumentation for Astronomy VIII, SPIE Astronomical Telescopes Instrumentation (2020)

[7] Kubo, M., et al., "SUNRISE Chromospheric Infrared spectroPolarimeter (SCIP) for SUNRISE III: Polarization Modulation Unit", Ground-based and Airborne Instrumentation for Astronomy VIII, SPIE Astronomical Telescopes Instrumentation (2020)

[8] Gandorfer, A., et al., "The Filter Imager SuFI and the Image Stabilization and Light Distribution System ISLiD of the Sunrise Balloon-Borne Observatory: Instrument Description”, Solar Physics 268, 35-55 (Jan. 2011).

[9] Tsuzuki, T., et al., "SunRISE Chromospheric Infrared spectroPolarimeter (SCIP) for SuNRISE III: Optical Design and Performance", Ground-based and Airborne Instrumentation for Astronomy VIII, SPIE Astronomical Telescopes Instrumentation (2020) 